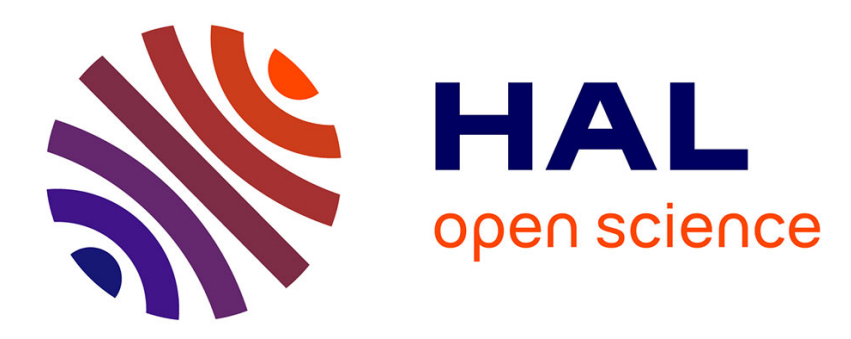

\title{
Viscosity scaling in hydrodynamic instabilities in porous media
}

Satyajit Pramanik

\section{To cite this version:}

Satyajit Pramanik. Viscosity scaling in hydrodynamic instabilities in porous media. CS-DC'15 World e-conference, Sep 2015, Tempe, United States. hal-01291086

\section{HAL Id: hal-01291086 \\ https://hal.science/hal-01291086}

Submitted on 20 Mar 2016

HAL is a multi-disciplinary open access archive for the deposit and dissemination of scientific research documents, whether they are published or not. The documents may come from teaching and research institutions in France or abroad, or from public or private research centers.
L'archive ouverte pluridisciplinaire HAL, est destinée au dépôt et à la diffusion de documents scientifiques de niveau recherche, publiés ou non, émanant des établissements d'enseignement et de recherche français ou étrangers, des laboratoires publics ou privés. 


\title{
Viscosity scaling in hydrodynamic instabilities in porous media
}

\author{
Satyajit Pramanik \\ Department of Mathematics \\ Indian Institute of Technology Ropar, Rupnagar 140001, Punjab, India
}

\begin{abstract}
The importance of viscosity scaling in the context of viscous fingering in a finite slice with viscosity dependent diffusivity is investigated theoretically. Choosing the characteristic viscosity classically as either the displacing or displaced fluid viscosity for both more and less viscous slice leads to inappropriate theoretical predictions, which do not support the physics. With an appropriate choice of the characteristic viscosity, we show that the onset of instability and the initial dynamics of the finger patterns are the same for both more and less viscous slices. Our analysis will be helpful in the theoretical understanding of buoyancydriven convection in a variable viscosity layer in vertical porous media or $\mathrm{VF}$ with non-monotonic viscosity profiles.
\end{abstract}

\section{Introduction}

Viscous fingering (VF) in various fluid flow problems of industrial and environmental interest, such as oil recovery [1], pollution remediation [2], $\mathrm{CO}_{2}$ sequestration [3], chromatography separation [4-7], etc. have drawn attention of many researchers over many decades. This hydrodynamic instability is featured when a less viscous fluid displaces a more viscous and hence less mobile one in porous media [1]. Based on the geometry of the interface featuring the instability in the form of finger-like structure, VF problems can be broadly classified into two categories. The first one being the classical Saffman-Taylor instability in two semi-infinite fluids separated by a flat interface [1], while the other is the displacement of a finite sample, typically in chromatography column [4]. In the latter case, the sample is confined in a rectangular region that can feature VF either at the frontal or rear interface depending on the viscosity contrast in the downstream direction. The influence of positive and negative log-mobility ratio was investigated in the context of solute adsorption on porous matrix [8]. In the absence of adsorption and for constant diffusivity of the solute concentration, Mishra et al. showed that the onset of instability and the subsequent dynamics of the fingers are identical for more and less viscous slices [9]. Pramanik and Mishra [12] showed that in the presence of the Korteweg stress $[10,11]$ the fingering dynamics of a miscible slice of more viscosity are identical to that of a less viscosity. This was achieved with an appropriate scaling. In this paper, we are interested to explore whether such dynamics are also possible with concentration dependent diffusivity in absence of the Korteweg stress effect. In this context, 
we propose a modified viscosity scaling that helps to answer this question when the diffusivity is inversely proportional to the viscosity of the fluid. We structure this paper as follows. Mathematical formulation and numerical solution of the present problem is discussed in Sect. 2, followed by result discussion and conclusion in Sects. 3 and 4, respectively.

\section{Mathematical formulation and numerical solution of the problem}

Consider a rectilinear displacement of a finite sample of viscosity $\mu_{2}$ by another fluid of viscosity $\mu_{1}$ at a uniform velocity $U e_{x}$, where $e_{x}$ is the unit vector in the $x$-increasing direction. The displacing fluid consists of the same sample solvent having no solute concentration in it, i.e. $c=0$, while the finite sample has solute concentration $c=c_{2}$. We assume the variation of the diffusion coefficient with concentration is governed by the generalized Stokes-Einstein relation $[13,14]$,

$$
D(c) \cdot \mu(c)=\text { constant }
$$

so that $D(c)=D_{0} / \mu(c)$, where $D_{0}$ denotes the diffusion coefficient of an infinitesimally small amount of the displaced fluid in the displacing fluid. Fluids are assumed to be incompressible, miscible and neutrally buoyant. For nondimensionalization we choose $D_{0} / U, D_{0} / U^{2}, U, \mu_{\mathrm{ch}} D_{0} / \kappa, \mu_{l}, c_{2}$ as the characteristic length, time, velocity, pressure, viscosity, and concentration, respectively [12]. Here $\mu_{l}=\min \left\{\mu_{1}, \mu_{2}\right\}, \kappa$ is the permeability of the porous media, and $D_{0}$ represents a reference diffusivity. The related dimensionless equations in a frame of reference moving with the velocity $U e_{x}$ can be written as [1],

$$
\nabla \cdot \boldsymbol{u}=0, \quad \nabla p=-\mu(c)\left(\boldsymbol{u}+e_{x}\right), \quad \frac{\partial c}{\partial t}+\boldsymbol{u} \cdot \nabla c=\nabla \cdot(D(c) \nabla c)
$$

where $D(c)$ represents concentration dependent dispersion. The viscosity of the fluids depends exponentially on the solute concentration, i.e., $\mu(c)=e^{R f(c)}$, where $R=\ln \left(\mu_{m} / \mu_{l}\right)$ is the $\log$-mobility ratio and $f(c)$ is $c$ (more viscous slice) or $1-c$ (less viscous slice), and $\mu_{m}=\max \left\{\mu_{1}, \mu_{2}\right\}$. Therefore, the dimensionless form of the concentration dependent viscosity becomes $D(c)=1 / \mu(c)$. For two dimensional incompressible flow we define the stream-function $\psi(x, y, t)$, such that $u=\psi_{y}, v=-\psi_{x}$ are the longitudinal and transverse component of the Darcy velocity $\boldsymbol{u}$. Therefore, we can write (2) as,

$$
\frac{\partial c}{\partial t}+J(x, y, t)=0, \quad \nabla^{2} \psi=-R f^{\prime}(c) N(x, y, t) .
$$

The nonlinear terms $J(x, y, t)$ and $N(x, y, t)$ in $(3)$ are defined as,

$$
\begin{aligned}
& J(x, y, t)=\psi_{y} c_{x}-\psi_{x} c_{y}-e^{-R f(c)}\left(\nabla^{2} c-R f^{\prime}(c)|\nabla c|^{2}\right), \\
& N(x, y, t)=\left(\nabla \psi+e_{y}\right) \cdot \nabla c
\end{aligned}
$$


where $e_{y}$ is the unit vector in $y$-direction.

Equations (3) are solved using a Fourier pseudo-spectral method $[15,4]$ to analyze the influence of variable diffusivity on VF of more and less viscous miscible slices. We apply Discrete Fourier transform (DFT) to $c(x, y, t), \psi(x, y, t), J(x, y, t)$ and $N(x, y, t)$ that converts (3) into differential algebraic equations,

$$
\frac{\mathrm{d} \hat{c}_{m, n}(t)}{2} \hat{J}_{m, n}(t)=0, \quad \hat{\psi}_{m, n}(t)=\hat{N}_{m, n}(t) /\left(k_{m}^{2}+k_{n}^{2}\right),
$$

Fig. 1. Spatial stittcture of the solute concentration at $t=800$ in the moving frame of

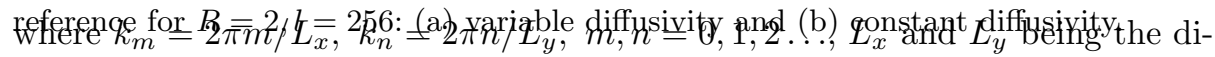
mensionless length and width of the computational domain, respectively. Equation (6) is solved using Adams-Bashforth predictor and trapezoidal corrector method in the Fourier space to obtain the Fourier modes at next time step $t+\Delta t$. Inverse DFT has been performed for $\hat{c}_{m, n}$ and $\hat{\psi}_{m, n}$ to update the con-

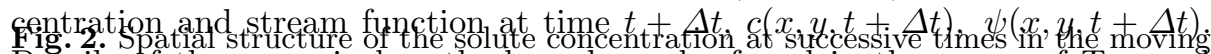

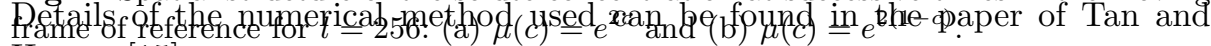
Homsy [15].

\section{Results and discussion}

First we investigate the influence of variable diffusivity on miscible VF in a finite slice. The spatial structure of a more viscous slice of dimensionless width $l=256$ is presented for variable diffusivity (Fig. 1a) and constant diffusivity (Fig. 1b) with $R=2$. Figure 1 depicts that the fingering dynamics become more complex for variable diffusivity as compared to constant diffusivity case. In the former case the wavelength of the fingers becomes shorter and the fingers propagate faster, which is readily evident from an early interaction of the fingers with the frontal interface.

Figure 2 shows the spatio-temporal evolution of the solute concentration of more and less viscous slice for $R=2, l=256$ with concentration dependent diffusivity. This figure depicts that, before interacting with the stable interface the fingering dynamics in more and less viscous slices are identical. We also perform simulations for a less viscous slice by considering $\mu_{\mathrm{ch}}=\mu_{1}[9]$, such that $\mu=e^{-2 c}$. It is observed that the finger patterns differ significantly in this case (for brevity not shown) as compared to that shown in Fig. 2b. In particular, the onset of instability delays when choosing $\mu=e^{-2 c}$, which is attributed to the fast diffusion of the solute concentration as compared to $\mu=e^{2(1-c)}$. Thus we conclude, in order to compare between two similar flows one should be careful about the choice of the dimensionless values in such a way that they represent the same dimensional values. With the prescribed scaling of the viscosity of the fluids this has been achieved in the case of variable diffusivity VF in finite slice.

The temporal evolution of degree of mixing $\chi(t)[16]$ has been investigated for both more and less viscous finite sample of width $l=256$. We mentioned in $\S 2$ that the displacement of a more or less viscous finite sample is represented by $\mu(c)=e^{R c}$ or $\mu(c)=e^{R(1-c)}$, respectively, where the log-mobility ratio $R>0$. 
Fig. 3. Temporal evolution of the degree of mixing for $l=256$ with $\mu(c)=e^{2 c}$ (dashdotted line), $\mu(c)=e^{2(1-c)}$ (continuous line) and $\mu(c)=e^{-2 c}$ (dashed line). Inset images show the concentration distribution for the last two cases at $t=500$ and 1500 .

Figure 3 depicts that the temporal evolution of the degree of mixing, $\chi(t)$, for these two cases are almost identical, even after the interaction of the fingers with the respective stable interface. The displacement of a less viscous finite sample can alternatively be represented by $\mu(c)=e^{R c}, R<0$ [9]. It is shown that at early times, say at $t=500$ in Fig. 3, mixing due to diffusion for $R<0$ is more than the mixing due to VF for $R>0$. However, at later times, for example at $t=1500, \mathrm{VF}$ enhances the fluid-fluid interface and hence the degree of mixing becomes larger for $R>0$ than its $R<0$ counterpart. This is due to the fact that the dimensionless diffusion coefficient, which depends on the local solute concentration, $c$. For $R=2$, the dimensionless diffusion coefficient is $e^{-2(1-c)}$, while for $R=-2$, the dimensionless diffusion coefficient is $e^{2 c}$. Therefore, when the displacement of a less viscous finite sample is represented by $\mu(c)=e^{-2 c}$, the finite slice diffuses faster than that when the displacement is represented by $\mu(c)=e^{R(1-c)}$. This leads to a faster diffusive mixing for $\mu(c)=e^{-2 c}$ than that for $\mu(c)=e^{2 c}$.

\section{Conclusion}

The influence of viscosity scaling on miscible VF in a finite slice with viscosity dependent diffusivity is investigated through numerical simulations. Choosing an appropriate characteristic viscosity we show that the fingering dynamics of a more viscous slice is identical to that of a less viscous one. We believe, the present scaling analysis shall be important to investigate the stability of a variable viscosity buoyantly unstable layer in vertical porous media [17] or miscible VF with non-monotonic viscosity profiles [18], and pave new way to understand fluid mixing in porous media.

\section{Acknowledgement}

Author is also grateful to Manoranjan Mishra for fruitful discussion and careful reading of the manuscript. Author gratefully acknowledges the financial support from the National Board for Higher Mathematics, Department of Atomic Energy, Government of India through a Ph.D. fellowship.

\section{Scientific validation}

This paper has been unanimously validated in a collaborative review mode with the following reviewers:

- Aǵota Tóth, University of Szeged,

- Sarah Klein, Université Paris-Sud. 


\section{References}

1. Homsy, G. M.: Viscous fingering in porous media. Annu. Rev. Fluid Mech. 19, 271311 (1987).

2. Welty, C., Kane III, A. C., Kauffman, L. J.: Stochastic analysis of transverse dispersion in density-coupled transport in aquifers. Water Resour. Research 39, 1150 (2003).

3. Huppert, H. E., Neufeld, J. A.: The fluid mechanics of carbon dioxide sequestration. Annu. Rev. Fluid Mech. 46, 255-272 (2014).

4. De Wit, A., Bertho, Y., Martin, M.: Viscous fingering of miscible slices. Phys. Fluids 17, 054114 (2005).

5. Mishra, M., Martin, M., De Wit A.: Influence of miscible viscous fingering of finite slices on an adsorbed solute dynamics. Phys. Fluids 21, 083101 (2009).

6. Rana, C., Martin, M., De Wit A., Mishra, M.: Combined influences of viscous fingering and solvent effect on the distribution of adsorbed solutes in porous media. RSC Adv. 4, 34369-34381 (2014).

7. Rana, C., Mishra, M.: Fingering dynamics on the adsorbed solute with influence of less viscous and strong sample solvent. J. Chem. Phys. 141, 214701 (2014).

8. Mishra, M., Martin, M., De Wit A.: Influence of miscible viscous fingering with negative log-mobility ratio on spreading of adsorbed analytes. Chem. Eng. Sci. 65, 2392-2398 (2010).

9. Mishra, M., Martin, M., De Wit A.: Differences in miscible viscous fingering of finite width slices with positive or negative log mobility ratio. Phys. Rev. E 78, 066306 (2008).

10. Pojman, P. A., Whitmore, C., Turco Liveri, M. L., Lombardo, R., Marszalek, J., Parker, R., Zoltowski, B.: Evidence of an effective interfacial tension between miscible fluids: Isobutyric acid-water and 1-butanol-water in a spinning drop tensiometer. Langmuir 22, 2569-2577 (2006).

11. Chen, C.-Y., Wang, L., Meiburg, E.: Miscible droplets in a porous medium and the effects of Korteweg stresses. Phys. Fluids 13, 2447-2456 (2001).

12. Pramanik, S., Mishra, M.: Viscosity scaling of fingering instability in finite slices with Korteweg stress. Europhys. Lett. 109, 64001 (2015).

13. Probstein, R. F.: Physicochemical hydrodynamics. John Wiley \& Sons, New Jersey (2003).

14. Sahu, K. C.: Double diffusive effects on pressure-driven miscible channel flow: Influence of variable diffusivity. Int. J. Multiphase Flow 55, 24-31 (2013).

15. Tan, C. T., Homsy, G. M.: Simulation of non-linear viscous fingering in miscible displacement. Phys. Fluids 31, 1330 (1988).

16. Jha, B., Cueto-Felgueroso, L., Juanes, R.: Fluid mixing from viscous fingering. Phys. Rev. Lett. 106, 194502 (2011).

17. Manickam, O., Homsy, G. M.: Fingering instabilities in vertical miscible displacement flow in porous media. J. Fluid Mech. 288, 75-102 (1995).

18. Manickam, O., Homsy, G. M.: Stability of miscible displacements in porous media with nonmonotonic viscosity profiles. Phys. Fluids, 5(6) 1356-1367 (1993). 